\title{
LETTER
}

\section{What size tube doctor? Bigger may be better - at least for weaning}

\author{
David JP O'Callaghan and Duncan Wyncoll*
}

Recent experience has compelled us to write this letter as several patients, referred to our hospital and labelled slow-weaners, made rapid progress when upsized to a larger internal diameter (ID) tracheostomy. Accelerated weaning with larger ID tracheostomies is partially explained by physical principles.

Flow through the airways (natural and artificial) is both turbulent and laminar. Flow through a tracheostomy/ endotracheal tube (ETT) is mainly laminar and governed by the Hagen-Poiseuille equation:

\section{Flow $=$ pressure $\times$ radius $^{4} \times \pi /$ length $\times$ viscosity $\times 8$}

The radius of a tube has the predominant influence on gas flow; small changes in tracheostomy/ETT ID can exert a large effect on flow. For a given pressure gradient, the patient will receive greater gas flow through a larger tracheostomy/ETT, reducing work of breathing (WoB). This may result in a more rapid weaning process than would be the case with a smaller ID tube.

Work performed in the 1960s demonstrated that WoB increases as tracheostomy ID reduces, and WoB through a tracheostomy is only less than WoB through the mouth with ID $\geq 10 \mathrm{~mm}$ [1]. Removable inner cannulae further reduce IDs by about 1 to $1.5 \mathrm{~mm}$ [2], and removing them reduces WoB and may aid weaning [3] - but with the attendant risk of tube occlusion. Respiratory secretions and/or biofilm may further reduce the effective ID.

Applying the Hagen-Poiseuille equation indicates that approximately $70 \%$ greater $\mathrm{WoB}$ may be required when a size $7 \mathrm{~mm}$ ID tube is used over a size $8 \mathrm{~mm}$ tube. WoB imposed by a tracheostomy is less than that imposed by an equivalent ID ETT [4], and one would rarely try to wean an adult patient with a $5.5 \mathrm{~mm}$ ID ETT in place; yet this sub-optimal scenario is not that dissimilar from using a $7 \mathrm{~mm}$ ID tracheostomy with an inner cannula (often reducing effective ID to $\sim 5.5 \mathrm{~mm}$ ). It is interesting

*Correspondence: duncan.wyncoll@gstt.nhs.uk

Department of Adult Critical Care, Guy's and St Thomas' NHS Foundation Trust, St Thomas' Hospital, 1st Floor East Wing, Lambeth Palace Road, London SE1 7EH, UK and surprising to note that, in large trials of early versus late tracheostomy, the size of the tubes used is not mentioned [5].

We contend that it should be standard practice in the majority of adult patients for both ETT and tracheostomy IDs to be at least $8 \mathrm{~mm}$ in females and $9 \mathrm{~mm}$ in males. Consideration should also be given to other strategies that maximise tracheostomy/ETT ID in the weaning phase. This attention should ensure that WoB is minimised during the weaning process, potentially reducing the time spent ventilated, especially amongst patients with limited respiratory reserve.

\section{Abbreviations}

ETT, endotracheal tube; ID, internal diameter; WoB, work of breathing.

\section{Competing interests}

DJPOC declares that he has no competing interests. DW has given paid lectures or consulted for Kimberly-Clark (Irving, TX, USA), Covidien (Mansfield, MA, USA), ConvaTec (Skillman, NJ, USA), Iskus Health (Dublin, Ireland), Sage Products (Cary, IL, USA), Eli Lilly and Company (Indianapolis, IN, USA), Pfizer Inc. (New York, NY, USA), Smiths (Portex) (Ashford, UK), Astellas (Chertsey, UK), Baxter (Louisville, KY, USA) and Johnson \& Johnson (New Brunswick, NJ, USA) and has a stock interest in Biovo Technologies (Tel Aviv, Israel). The authors declare that they have no personal financial interests.

\section{Published: 27 March 2013}

\section{References}

1. Garzon AA, Seltzer B, Lichtenstein S, Karlson KE: Influence of tracheostomy cannula size on work of breathing. Ann Surg 1965, 162:315-320.

2. Hess DR: Tracheostomy tubes and related appliances. Respir Care 2005, 50:497-510.

3. Carter A, Fletcher SJ, Tuffin R: The effect of inner tube placement on resistance and work of breathing through tracheostomy tubes: a bench test. Anaesthesia 2013, 68:276-282.

4. Pierson DJ: Tracheostomy and weaning. Respir Care 2005, 50:526-533.

5. Terragni PP, Antonelli M, Fumagalli R, Faggiano C, Berardino M, Pallavicini FB, Miletto A, Mangione S, Sinardi AU, Pastorelli M, Vivaldi N, Pasetto A, Della Rocca G, Urbino R, Filippini C, Pagano E, Evangelista A, Ciccone G, Mascia L, Ranieri VM: Early vs late tracheotomy for prevention of pneumonia in mechanically ventilated adult ICU patients: a randomized controlled trial. JAMA 2010, 303:1483-1489.

doi:10.1186/cc12545

Cite this article as: O'Callaghan DJP, Wyncoll D: What size tube doctor? Bigger may be better - at least for weaning. Critical Care 2013, 17:422. 